\title{
Cytotoxic-free treatment designs: time to resistance
}

\author{
Justin Stebbing and Tim Crook
}

The most crystalline answers in biology have resulted from an understanding of the relationship between structure and function. Nowhere is this more apparent than in the statement in Watson and Crick's seminal paper that "It has not escaped our notice that the specific pairing we have postulated immediately suggests a possible copying mechanism for the genetic material" (Watson JD and Crick FHC [1953] Nature 171: 737). Such study theoretically offers a panacea for potential drug design and affords scientists and physicians the opportunity to achieve an appropriate patient-centric balance between efficacy and toxicity. Today, the most exploited proof-of-principle example of such an achievement in everyday oncology practice is the bench to bedside inhibition of various members of the EGFR family, including HER2. As a result, an impressive array of therapies have been developed, including agents that bind to various domains of the transmembrane receptor tyrosine kinases - notably the monoclonal antibody trastuzumab-and the small-molecule EGFR inhibitors gefitinib and erlotinib. Other agents include those that inhibit the activity of the non-receptor tyrosine kinases, such as imatinib, nilotinib and dasatanib. All of these therapies directly inhibit the catalytic activity of the kinase by interfering with the binding of ATP or substrates, or blocking dimerization and ligand binding.

Considering the multiplicity of signaling in cancer cells and the redundancy of a bewildering array of pathways, it can be of no surprise that, for the most part, inhibition of an individual kinase will not cure cancer. Indeed, apart from dramatic responses in hematologic malignancies and gastrointestinal stromal tumors, only one randomized monotherapy study has shown that the small-molecule inhibitors increase survival (Shepherd FA et al. [2005] N Engl J Med 353: 123-132). The situation is complicated, however, by the realization that specific molecular genetic changes are frequently present in the target macromolecule. In non-small-cell lung cancer, individuals with gain-of-function somatic
Overcoming

and measuring

resistance

to agents as

diverse as

tamoxifen and

imatinib....will

be more than

an incremental

step on a path

to curing cancer

\section{JStebbing is}

Consultant Medical

Oncologist and

Senior Lecturer at

the Department of

Medical Oncology,

Charing Cross and

Hammersmith

Hospitals, and T Crook

is a specialist registrar

in Medical Oncology

at the Department of

Medical Oncology,

Charing Cross and

Hammersmith

Hospitals, and

Team Leader in

the Laboratory of

Cancer Genetics and

Epigenetics at the

Breakthrough Breast

Cancer Research

Centre, Institute

of Cancer Research,

London, UK.

\section{Competing interests}

The authors declared

they have no competing

interests.

www.nature.com/clinicalpractice doi:10.1038/ncponc0695 mutations in the EGFR could be expected to have a poorer response to therapy, yet, paradoxically, the same individuals may be those most likely to respond to erlotinib. A comparable situation exists in breast cancer, in which overexpression of HER2 is associated with a more aggressive clinical phenotype, yet confers sensitivity to trastuzumab. Thus, the presence of mutations, or overexpression, becomes predictive of response. Unlike c-KIT mutations in gastrointestinal stromal tumors, such mutations do not cause constitutive activation but appear to enhance the activity of the receptor to its ligand (i.e. EGF or an inhibitory drug). A picture starts to emerge in which nefarious phosphorylated kinase pathways drive deregulated clonal cell proliferation, invasion and metastasis, whilst inhibition of these pathways using the right drugs at the right times in the right patients will probably disenfranchise the use of cell-cycle-specific and cell-cycle-nonspecific cytotoxics. As for triple therapy in HIV treatment (cost considerations aside), the right balance may end up being a combination of EGFR-binding drugs, at the same time and/or in sequence. Trials of combinations of imatinib and dasatanib are already underway, and the use of BCR-ABL transcripts or phosphorylation (of specific sites) as a measure of 'time to resistance' in clinical trials should now be entertained.

This issue has broader ramifications, including circumvention of ligand-independent autophosphorylation-induced activation of the estrogen receptor in breast cancer, a process clearly involving extensive cross-talk of multiple members of the EGFR family. Whilst there are multiple mechanisms of cross-talk, phosphorylated receptors represent a structure-function view of resistance-the only one we have. Overcoming and measuring resistance to agents as diverse as tamoxifen and imatinib, based on foundations of structure and function, will be more than an incremental step on a path to curing cancer. This process will probably have negative consequences for the conventional use of cytotoxics. 